\title{
Circulating Non-Coding RNA Biomarkers for Diabetes Mellitus
}

\author{
Gopinath Meenakshi Sundaram* \\ Agency for Science Technology \& Research, Institute of Medical Biology, Singapore
}

Submission: August 22, 2017; Published: August 29, 2017

*Corresponding author: Gopinath Meenakshi Sundaram, Agency for Science Technology \& Research (A*STAR), Institute of Medical Biology, Singapore, Tel: 6564070171; Email: Gopinath.Sundaram@imb.a-star.edu.sg

\begin{abstract}
Diabetes mellitus (DM) is a metabolic disease with a major health burden in both developed and developing countries. While type I DM is caused by destruction of pancreatic islets leading to decreased insulin secretion, type II DM is due to insulin resistance on the target cells, though both result in increased blood glucose levels. This has direct consequences on various tissues in the body leading to diabetes associated complications such as neuropathy, cardiovascular diseases, nephropathy and retinopathy. It is estimated nearly half the cases of DM are undiagnosed and early detection of DM in pre diabetes stage will have a great impact in precluding the above complications. Non-coding RNAs are emerging as novel players in both development and disease process. Many of them are secreted in bio-fluids and are currently evolving as excellent biomarkers for various diseases including cancer. In this review, I summarise some of the recent developments and discoveries made in the field of different types of non-coding RNAs as biomarkers for DM. With the current state of knowledge, it is tempting to speculate that noncoding RNAs may occupy the standard diagnostic procedures in clinics for early detection of DM in near future.
\end{abstract}

Keywords: Non-coding RNAs; Micro RNAs; Long non-coding RNAs; Diabetes

\section{Introduction}

The word Diabetes Mellitus (DM) was coined by the English Physician Thomas Willis in 1674 which was formerly known as "Willis's Disease". DM has been described from 1st century B.C.E. Diabetes in Greek means "siphon" referring to the excessive urination associated with this disease and mellitus referring to "like honey" indicates the presence of sugar (glycosuria) in urine. This is due to the increased glucose levels in blood leading to several other severe complications such as cardiovascular diseases, diabetic retinopathy, diabetic nephropathy and diabetic neuropathy. Subsequently, in the year 1683, Johann Conrad Brunner, a Swiss anatomist is the first one to demonstrate that elimination of pancrease from Dogs led to glycosuria suggesting a direct link between insulin and DM. Four centuries later, DM is rampant across the globe with more than 400 million diabetes cases worldwide and its expected rise to 600 million by 2040 . At least 5 million people die annually due to diabetes in which diabetes associated cardiovascular complications being one of the major causes while blindness (retinopathy), neuropathy and end stage renal disease (ESRD) are also additional disease burdens caused by DM. In 2015, the expenditure on treating diabetic patients exceeded 600 billion USD in US alone (source:
International Diabetic Federation). Diabetes is classified into two major groups. Type I diabetes (T1DM), otherwise known as juvenile diabetes or insulin dependent, in which insulin producing pancreatic- $\beta$ cells are destroyed due to auto immune response. T1DM is well controlled by exogenous administration of insulin. Type II diabetes (T2DM), which constitutes $90 \%$ of diabetic cases worldwide is associated with insulin resistance and decreased insulin secretion by pancreatic $\beta$-cells. Unlike T1DM which has a sudden onset and is detected early, T2DM develops gradually and patients are usually unaware of this disease until secondary complications arise. Hence, early detection of T2DM is a critical factor to determine the curability of the disease. In this review, I summarise the recent literature pertaining to circulating non-coding RNAs as biomarkers for DM. Even though the vast majority of molecular mechanisms of DM with reference to protein coding genes has been investigated through past decades, non-coding RNAs are gaining particular attention as both therapeutic targets and biomarkers in the field. This is particularly applicable since most genetic variations associated with diabetes are located in non-protein coding regions of the genome [1]. 


\section{Current Research in Diabetes \& Obesity Journal}

\section{Non-coding RNAs: origin, classification and function}

With the advent of high throughput next-generation sequencing at an affordable cost, the past decade has seen a dramatic increase in attention on the non-coding portion of the genome, a region which was considered as genomic junk or noise earlier. Non-coding RNAs, as the name suggest do not encode proteins and constitute more than $70 \%$ of genomic transcriptional output (For a detailed review see Palazzo and Lee [2]). They are divided broadly into the classical non-coding RNAs discovered many decades ago, such as transfer RNAs, ribosomal RNAs and nuclear/nucleolar RNAs and the nonclassical non-coding RNAs discovered in the past two decades being the short non-coding RNAs (less than 200nt) comprising micro RNAs (miRNAs), piwi interacting RNAs (piRNAs), long non-coding RNAs (lncRNAs) and circular RNAs which are longer than 200nt in length $[3,4]$. In general, the function of non-coding RNAs appears to regulate the transcription/stability/translation of coding mRNAs at multiple layers. Micro RNAs are known to contain seed sequences for target mRNAs and the direct binding of miRNAs to the target mRNAs generally leads to target mRNA translational repression and/or transcript degradation. On the other hand, IncRNAs employ a wide variety of mechanisms in regulating the proteome output of the cell such as recruitment of transcription factors and epigenetic modifiers to protein coding genes, regulation of mRNA stability by directly binding to target mRNAs or by inhibiting miRNAs by their sponging activity, acting as scaffold for proteins and as enhancer/antisense transcripts for neighbouring/distant genes [5]. Circular RNAs (circRNAs) are an emerging sub-class of lncRNAs, derived by back-splicing of precursor mRNAs, act as miRNAs sponges with the current state of knowledge $[6,7]$. Two decades ago, $\mathrm{X}$ inactivation centre transcript (XIST) is the first lncRNAs identified with a defined function in $\mathrm{X}$ chromosome inactivation in female somatic cells [8]. Now as per databases cataloguing non-coding RNAs, there are roughly 147,000 annotated human IncRNAs (source: lncipedia.org), 2500 mature human miRNAs (source: mirbase. org) and thousands of circRNAs (source: circbase.org) indicating an unexplored territory of molecules relevant to human diseases. There are abundant reports on the role of miRNAs/lncRNAs pertinent to DM or DM related complications and are reviewed elsewhere [9-11].

\section{Circulating miRNAs in DM}

The remarkable stability of miRNAs in body fluids makes them an ideal and robust candidates for biomarkers of diseases [12]. Micro RNAs have been detected in almost all human bio fluids and show fluid specific profile patterns [13]. Presence of miRNAs in serum samples were first described Chen and Colleagues [14]. In this report, the authors identified 190 known miRNAs in serum samples and also demonstrated the stability and RNAs resistance of miRNAs in serum. miR-375 was initially identified as pancreatic islet specific miRNA [15]. In addition to its role in pancreatic development [16], it also regulates insulin secretion from pancreatic $\beta$-cells by targeting Myotrophin. miR375 was also detected serum samples of mice injected with Streptozotocin to induce diabetes and non-obese diabetic (NOD) mice with chronic hyper-glycaemia [17].

Recently, in addition to miR-375, Higuchi et al also identified miR-101 and miR-802 as circulating biomarkers in a cohort of 150 T2DM patients compared to healthy controls [18]. Endothelial dysfunction directly contributes to the development of atherosclerosis and cardiovascular diseases, which are tightly linked to DM. It is generally agreed that hyperglycaemia and DM leads to endothelial dysfunction. In an effort to identify circulating miRNAs specific to T2DM, miR-126, a miRNA enriched in endothelial cells and critical for its function, was found to be down regulated in plasma from T2DM compared to healthy controls [19]. This was the first large scale study identifying a blood miRNA signature for T2DM with more than 800 patients. In addition, miR-661, miR-571, miR-770-5p, miR-892b and miR1303 have recently been identified as circulating biomarkers for T2DM associated micro vascular complications [20].

Transforming growth factor- $\beta$ (TGF- $\beta$ ) signalling is a critical mediator of fibrosis in renal tissues leading to renal failure. In DM patients, high blood glucose levels stimulate the increased renal secretion of TGF- $\beta$ resulting in fibrosis [21]. A separate study with a well-defined cohort of T1DM patients, Pezzolesi et al. [22] identified four miRNAs in plasma with a direct relevance to patients progressing to ESRD and non-progressors. Especially TGF-B regulated miRNAs such as miR-29a-3p and let-7c-5p were found to be specifically down regulated and miR-21-5p and let$7 \mathrm{~b}-5 \mathrm{p}$ was specifically up regulated in patients progressing to ESRD compared to non-progressing group. This is in line with the function of miR-29a-3p/let-7c-5p which negatively fibrotic genes while miR-21-5p/let-7b-5p which is functionally associated with renal dysfunction [22].

Early stratification of patients with insulin resistance (T2DM) to treatment with insulin sensitizing agents such as Thiazolidinedione's (TZD) determines the right kind of treatment options. In this line, Flowers et al. [23] measured a panel of 25 circulating miRNAs with a known role in glucose/insulin metabolism in individuals responding or not responding to TZD treatment and found miR-320a and miR486 as a biomarker for TZD responders [23]. Similarly, using next generation sequencing, Shah et al. [24] profiled circulating miRNAs in serum samples of 2,317 non-diabetic participants and identified 16 miRNAs that are associated with insulin resistance (IR). In particular, miR-122 was closely associated with insulin resistances as well adiposity in adults [24]. The literature describing circulating miRNAs as biomarkers for DM is currently expanding at an exponential phase [25-27]. Moreover, in an integrated meta-analysis of 59 independent studies focussing on miRNA biomarkers in T2DM, He and colleagues extracted 158 deregulated miRNAs in T2DM, with specifically differential expression patterns across 7 major tissue types which included whole blood, plasma and serum in 
which 16 miRNAs shared the same profile in plasma and serum [28]. These miRNAs may form the basis of future research with respect to their targets and mechanism of action which forms basic criteria to be a suitable biomarker.

\section{Circulating IncRNAs in DM}

Based on their origin from the genome, IncRNAs are broadly classified into intergenic, intronic, anti-sense, divergent and enhancer derived lncRNAs. Keeping in view that both T1DM and T2DM have pancreatic $\beta$-cell dysfunction, identification of IncRNAs specific for this cell type will offer targeted therapies. In this line, Moran et al. [29], performed a systematic profiling of the global lncRNA expression in human pancreatic islets and $\beta$-cells using RNA-sequencing approach. In this study, thousands of IncRNAs were found to be expressed in human pancreatic islets and many of them showed specific expression in $\beta$-cell.

Notably, a significant fraction of these lncRNAs mapped closer to protein coding genes involved in $\beta$-cell function. A growing body of evidence indicate the critical function of numerous lncRNAs in regulating glucose homeostasis, insulin metabolism and diabetes related complications [30-32]. Interestingly, the first circular RNA function related to DM was CiRS-7. CiRS-7 has more than 50 binding sites for miR-7 was shown as a competing endogenous RNA for miR-7 which inhibits insulin secretion. Over expression of CiRS-7 inhibits miR-7 mediated repression of insulin secretion [33].

However, only a few reports have discussed the potential of circulating lncRNAs in DM or its associated complications. Growth-arrest specific transcript 5 (GAS5) is a IncRNA which has a proven role in regulating cell growth and survival by modulating mTOR signalling pathway in T-lymphocytes. In a recent study comparing lncRNA expression profiles in serum samples of diabetic $(n=47)$ vs. non-diabetic $(n=49)$ patients, GAS5 was markedly down regulated in the former samples [34]. Particularly, GAS5 levels were down regulated in patients with HbA1c $>5.9$, who were not categorized as diabetic (due to the clinical cut off for T2DM being HbA1c>6.5) indicating GAS5 can be a predictive biomarker for T2DM.

Another interesting result from this study is the lack of differential expression of IncRNAs in patient serum samples, known to be involved in insulin secretion/glucose metabolism such as ANRIL, H19 and HOTAIR, indicating that serum IncRNAs may not necessarily correlate with tissue IncRNA expression profiles. As an additional layer of complication, circulating long intergenic non-coding RNA predicting cardiac remodelling (LIPCAR) was found to be an independent predictor of left ventricular diastolic function in T2DM patients, while Myocardial infarction-associated transcript (MIAT) and smooth muscle and endothelial cell-enriched migration/differentiationassociated long non-coding RNA (SENCR) were found to be predictive biomarkers for ventricular remodelling in T2DM patients, though these transcripts per se do not show differential expression in non-diabetic versus diabetic patient serum [35].
Nevertheless, SENCR is the first circulating lncRNA whose serum expression levels were shown to predict responsiveness to pioglitazone therapy (to improve insulin sensitivity and left ventricular diastolic function) in T2DM patients [36]. Until now, very few studies have investigated the potential of circulating lncRNAs as biomarkers for DM or DM associated complications.

\section{Conclusion}

Circulating non-coding RNAs are better alternative to conventional protein based biomarkers for multiple reasons:

A) The robust stability of non-coding RNAs in multiple body fluids including whole blood/plasma/serum, saliva and urine which are easily obtainable with minimally-invasive methods [13,37].

B) RNA based detection methods works at a much better sensitivity range (in picograms) than protein based detection methods (nano grams) and the specificity of RNA detection is far superior than proteins and C) The current methods for testing T1DM involve measuring the serum auto antibodies for pancreatic $\beta$-cells or C-peptides derived from endogenous insulin and However, these methods are expensive and time intensive, while RNA based detection methods will comparatively be cost effective in future.

Nevertheless, certain major concerns need to be addressed before non-coding RNAs are implemented as biomarkers in clinical settings. Firstly, though miRNAs show excellent nuclease resistant in serum samples, the absolute levels of miRNAs are confounded by other miRNAs that are resultant of haemolysis which can significantly influence the proportion of target miRNAs in sample preparation [38]. Secondly, in many instances, the origin of these non-coding RNAs in body fluids is still unclear and only a handful of non-coding RNAs show direct correlation with the changes in pancreatic cells in DM [25].

Thirdly, unlike cellular RNA, there is no consensus normalization controls for serum miRNAs with spike in controls and endogenous controls have been used in different studies. In addition, the levels of endogenous control miRNAs can also be affected by the quality of RNA isolated and the methodology used [39]. Lastly, a recent report indicate majority of circulating lncRNAs show much lower transcript abundance compared to their corresponding tissue of origin [40]. In summary, noncoding RNAs are promising candidates as predictive biomarker for diseases and the future research will achieve significant progress in solving these issues.

\section{References}

1. Cebola I, Pasquali L (2015) Non-coding genome functions in diabetes. Journal of Molecular Endocrinology 56(1): 1-20.

2. Palazzo AF, Lee ES (2015) Non-coding rna: what is functional and what is junk? Front Genet 6: 2.

3. St Laurent G, Wahlestedt C2, Kapranov P (2015) The Landscape of long non coding rna classification. Trends in Genetics 31(5): 239-251.

4. Magdalena L, Kotlinowski J, Jura J (2016) Long noncoding rnas in 


\section{Current Research in Diabetes \& Obesity Journal}

metabolic syndrome related disorders. Mediators of Inflammation.

5. Fatica A, Bozzoni I (2014) Long non-coding RNAs: new players in cell differentiation and development. Nature Reviews Genetics 15(1): 7-21.

6. Chen LL (2016) The biogenesis and emerging roles of circular RNAs. Nature Reviews Molecular Cell Biology 17(4): 205-211.

7. Greene J, Baird AM, Brady L, Lim M, Gray SG, et al. (2017) Circular RNAs: biogenesis, function and role in human diseases. Front Mol Biosci 4: 38

8. Herzing, LB, Romer JT, Horn JM, Ashworth A (1997) Xist has properties of the x-chromosome inactivation centre. Nature 386(6622): 272-275.

9. Motterle A, Sanchez Parra C, Regazzi R (2016) Role of long noncoding rnas in the determination of $\beta$-cell identity. Diabetes Obesity Metabolism 18(supply 1): 41-50.

10. Fernandez Valverde SL, Taft RJ, Mattick JS (2011) Micro RNAs in betacell biology, insulin resistance, diabetes and its complications. Diabetes 60(7): 1825-1831.

11. Guay C, Roggli E, Nesca V, Jacovetti C, Regazzi R (2011) Diabetes Mellitus, a micro RNA-related disease? Translational Research 157(4): 253-264.

12. Turchinovich A, Weiz L, Langheinz A, Burwinkel B (2011) Characterization of extracellular circulating micro RNA. Nucleic Acids Research 39 (16): 7223-7233.

13. Weber JA, Baxter DH, Zhang S, Huang DY, Huang KH, et al. (2010) The micro RNA spectrum in 12 body fluids. Clinical Chemistry 56 (11): $1733-1741$

14. Chen X, Ba Y, Ma L, Cai X, Yin Y, et al. (2008) Characterization of micro RNAs in Serum: A novel class of biomarkers for Diagnosis of cancer and other diseases. Cell Research 18(10): 997-1006.

15. Poy MN, Eliasson L, Krutzfeldt J, Kuwajima S, Ma X, et al. (2004) A Pancreatic islet-specific micro rna regulates insulin secretion. Nature 432 (7014): 226-230

16. Li X (2014) MiR-375, a microRNA related to diabetes. Gene 533(1): 1-4

17. Erener S, Mojibian M, Fox JK, Denroche HC, Kieffer TJ (2013) Circulating miR-375 as a $\beta$-cell death and diabetes in mice. Endocrinology 154 (2) 603-608.

18. Higuchi C, Nakatsuka A, Eguchi J, Teshigawara S, Kanzaki M, et al. (2015) Identification of circulating miR-101, miR-375 and miR-802 as biomarkers for type 2 diabetes. Metabolism: Clinical and Experimental 64(4): 489-497.

19. Zampetaki A, Kiechl S, Drozdov I, Willeit P, Mayr U, et al. (2010) Plasma micro RNA profiling reveals loss of endothelial MiR-126 and other micro RNAs in Type 2 diabetes. Circulation Research 107(6): 810-817.

20. Wang C, Wan S, Yang T, Niu D, Zhang A, et al. (2016) Increased serum micro RNAs are closely associated with the presence of microvascular complications in type 2 diabetes mellitus. Scientific Reports pp. 1-9.

21. Sharma K, Ziyadeh FN, Alzahabi B, McGowan TA, Kapoor S, et al. (1997) Increased renal production of transforming growth factor-beta1 in patients with type II diabetes. Diabetes 46(5): 854-859.

22. Pezzolesi MG, Satake E, McDonnell KP, Major M, Smiles AM, et al. (2015) Circulating TGF-beta1-regulated miRNAs and the risk of rapid progression to ESRD in type 1 diabetes. Diabetes 64(9): 3285-3293.

23. Flowers E, Aouizerat BE, Abbasi F, Lamendola C, Grove KM, et al. (2015) Circulating microRNA-320a and microRNA-486 predict thiazolidinedione response: moving towards precision health for diabetes prevention. Metabolism: Clinical and Experimental 64(9): 1051-1059.
24. Shah R, Murthy V, Pacold M, Danielson K, Tanriverdi K, et al. (2017) Extracellular RNAs are associated with insulin resistance and metabolic phenotypes. Diabetes Care 40(4): 546-553.

25. Guay C, Regazzi R (2013) Circulating microRNAs as novel biomarkers for diabetes mellitus. Nat Rev Endocrinol 9(9): 513-521.

26. Chien HY, Lee TP, Chen CY, Chiu YH, Lin YC, et al. (2015) Circulating microRNA as a diagnostic marker in populations with type 2diabetes mellitus and diabetic complications. Journal of the Chinese Medical Association 78(4): 204-211

27. Simó Servat O, Simó R, Hernández C (2016) Circulating biomarkers of diabetic retinopathy: an overview based on physiopathology. Journal of Diabetes Research

28. Yuqing He, Yuanlin Ding, Biyu Liang, Juanjuan Lin, Taek Kyun Kim, et al. (2017) A Systematic study of dysregulated MicroRNA in type 2 diabetes mellitus. International Journal of Molecular Sciences 18(3): 456

29. Morán I, Akerman I, van de Bunt M, Xie R, Benazra M, et al. (2012) Human $\beta$ cell transcriptome analysis uncovers Incrnas that are tissuespecific, dynamically regulated, and abnormally expressed in type 2 diabetes. Cell Metabolism 16(4): 435-448.

30. Beltrami C, Angelini TG, Emanueli C (2015) Non coding RNAs in diabetes vascular complications. Journal of Molecular and Cellular Cardiology 89: 42-50.

31. Sun X, Wong D (2016) Long noncoding RNA mediated regulation of glucose homeostasis and diabetes. Am J Cardiovasc Dis 6(2): 17-25.

32. Aashiq HM, Simranjeet Kaur, Pociot F (2017) Long non-coding RNAs as novel players in $\beta$ cell function and type 1 diabetes. Human Genomics 11: 17.

33. Xu H, Guo S, Li W, Yu P (2015) The circular RNA Cdr1as, via miR-7 and its targets, regulates insulin transcription and secretion in islet cells. Scientific Reports 5: 12453.

34. Carter G, Miladinovic B, Patel AA, Deland L, Mastorides S, et al (2015) Circulating long noncoding RNA GAS5 levels are correlated to prevalence of type 2 diabetes mellitus. BBA Clinical 4: 102-117.

35. de Gonzalo Calvo D, Kenneweg F, Bang C, Toro R, van der Meer RW et al. (2016) Circulating long-non coding RNAs as biomarkers of left ventricular diastolic function and re modelling in patients with wellcontrolled type 2 diabetes. Scientific Reports 6: 37354.

36. de Gonzalo Calvo D, Kenneweg F, Bang C, Toro R, van der Meer RW, et al. (2016) Circulating long noncoding RNAs in personalized medicine. Journal of the American College of Cardiology 68(25): 2914-2916.

37. Shi T, Gao G, Cao Y (2016) Long noncoding RNAs as novel biomarkers have a promising future in cancer diagnostics. Disease Markers.

38. Ravi R, Tanriverdi K, Daniel L, Larson M, Gerstein M , et al. (2016) Discordant expression of circulating micro RNA from cellular and extracellular sources. PLoS ONE.

39. Marabita F, De Candia P, Torri A, Tegnér J, Abrignani S, et al. (2016) Normalization of circulating micro RNA expression data obtained by quantitative real-time RT-PCR. Briefings in Bioinformatics 17(2): 204212.

40. Schlosser K, Hanson J, Villeneuve PJ, Dimitroulakos J, McIntyre L, et al. (2016) Assessment of circulating Lnc RNAs under physiologic and pathologic conditions in humans reveals potential limitations as biomarkers. Sci Rep 6: 36596. 

(C) Commons Attribution 4.0 Licens

DOI: $10.19080 / C R D O J .2017 .3 .555622$
Your next submission with Juniper Publishers will reach you the below assets

- Quality Editorial service

- Swift Peer Review

- Reprints availability

- E-prints Service

- Manuscript Podcast for convenient understanding

- Global attainment for your research

- Manuscript accessibility in different formats ( Pdf, E-pub, Full Text, Audio)

- Unceasing customer service

Track the below URL for one-step submission https://juniperpublishers.com/online-submission.php 